\title{
(2) \\ (อ) OPEN ACCESS \\ How to iGuide: flat panel detector, CT-assisted, minimally invasive evacuation of intracranial hematomas
}

\author{
David Dornbos III @ (1, 1,2,3 Cathra Halabi, ${ }^{4,5}$ Julie DiNitto, ${ }^{6}$ Kerstin Mueller, ${ }^{6}$ \\ David Fiorella (1) , 7,8 Daniel L Cooke, ${ }^{9}$ Adam S Arthur (1) 2,3
}

- Additional supplemental material is published online only. To view, please visit the journal online (http://dx.doi. org/10.1136/neurintsurg2021-017903).

For numbered affiliations see end of article.

Correspondence to Dr Adam S Arthur, SemmesMurphey Neurologic and Spine Institute, Memphis, Tennessee, USA; aarthur@semmesmurphey.com

Received 10 June 2021 Accepted 19 September 2021

Check for updates

(c) Author(s) (or their employer(s)) 2021. Re-use permitted under CC BY-NC. No commercial re-use. See rights and permissions. Published by BMJ.

To cite: Dornbos III D, Halabi C, DiNitto J, et al. J Neurolntervent Surg Epub ahead of print: [please include Day Month Year]. doi:10.1136/

neurintsurg-2021-017903

\section{ABSTRACT}

Evidence is growing to support minimally invasive surgical evacuation of intraparenchymal hematomas, particularly those with minimal residual hematoma volumes following evacuation. To maximize the potential for neurologic recovery, it is imperative that the trajectory for access to the hematoma minimizes disruption of normal parenchyma. Flat panel detector CT-based navigation and needle guidance software provides a platform that uses flat panel detector CT imaging obtained on the angiography table to aid reliable and safe access to the hematoma. In addition to providing a high degree of accuracy, this method also allows convenient and rapid re-imaging to assess navigation accuracy and the degree of hematoma evacuation prior to procedural completion. We provide a practical review of the syngo iGuide needle guidance software and the methodology for incorporating its use, and the software of other vendors, in a variety of minimally invasive methods for evacuation of intraparenchymal hematomas.

\section{INTRODUCTION}

Primary spontaneous intracerebral hemorrhage (ICH) is associated with the highest morality rate among stroke subtypes, approaching 50\% at 1 year. ${ }^{12}$ Among patients who survive ICH, nearly $80 \%$ do not achieve functional independence. ${ }^{3}$ Evidence-based acute interventions aim to minimize hemorrhage enlargement and secondary brain injury via specialty medical management in an intensive care or dedicated stroke unit in order to monitor and treat clinical deterioration. Early clinical deterioration typically signals active hematoma expansion, elevated intracranial pressure from mass effect, hydrocephalus, herniation, seizure, or other emergent complications, that warrant swift and targeted treatment. ${ }^{4}$

Hemorrhage stabilization is lifesaving with a clear reduction in morbidity and mortality, and medical treatments include strict blood pressure control and coagulopathy reversal. Although medical management of ICH considerably mitigates hematoma expansion and secondary brain injury, it does not acutely reverse or reduce hematoma volume, perilesional edema, or any related structural, physiologic, or biochemical impacts on brain parenchyma from the hematoma itself. Moreover, large or increasing hematoma volumes portend a worsening prognosis. ${ }^{5}$ Procedural hematoma reduction or elimination is thus an appealing companion treatment to medical management, yet clinical benefit has been demonstrated only for surgical treatment of cerebellar hemorrhage (class I, level B evidence) for selective patients (ie, patients with hematoma $>3 \mathrm{~cm}$, obstructive hydrocephalus, and/or clinical deterioration). ${ }^{4}$ At this point, there are no proven acute $\mathrm{ICH}$ interventions analogous to those for vaso-occlusive acute ischemic stroke. ${ }^{6}$

Neurosurgical decompressive hemicraniectomy with or without hematoma evacuation for supratentorial $\mathrm{ICH}$ is typically a salvaging maneuver to prevent further clinical deterioration from lesion expansion, imminent brain herniation, and/or rise in refractory intracranial pressure. Open surgical hematoma evacuation for supratentorial $\mathrm{ICH}$ has not demonstrated benefit over medical therapy in the international Surgical Trial in Intracerebral Hemorrhage (STICH) series and related subgroup analyses. ${ }^{78}$ Results suggested that a targeted patient population (ie, those with hematoma within $1 \mathrm{~cm}$ of the cortical surface and no intraventricular extension) might benefit from neurosurgical hematoma evacuation, but patient and procedural heterogeneity and significant study group crossover limited interpretation in the study.

Minimally invasive surgery (MIS) can safely reduce hematoma volume in carefully selected patients. Numerous studies have reported safety and efficacy of MIS evacuation through multiple techniques, ${ }^{29-14}$ and several meta-analyses have demonstrated improved rates of dependency and mortality compared with conventional treatment and open surgical evacuation. ${ }^{9} 1516$ Subjects in the MIS treatment arm of the Minimally Invasive Surgery and Tissue Plasminogen Activator in ICH Evacuation (MISTIE) phase III study showed similar functional outcomes to those of subjects treated with medical management. However, fewer than two-thirds of patients undergoing MIS evacuation demonstrated the targeted post-treatment hematoma volume of $<15 \mathrm{~mL}$, and subsequent analyses suggested that subjects with post-surgical hematoma volumes of $<15 \mathrm{~mL}$ had better functional outcomes than subjects treated with medical management. ${ }^{17}$ These exploratory analyses should be interpreted with caution, but MIS seems to be a safer alternative than open neurosurgery with less manipulation of healthy or perilesional brain tissue. MIS techniques have continued to evolve and are currently used 

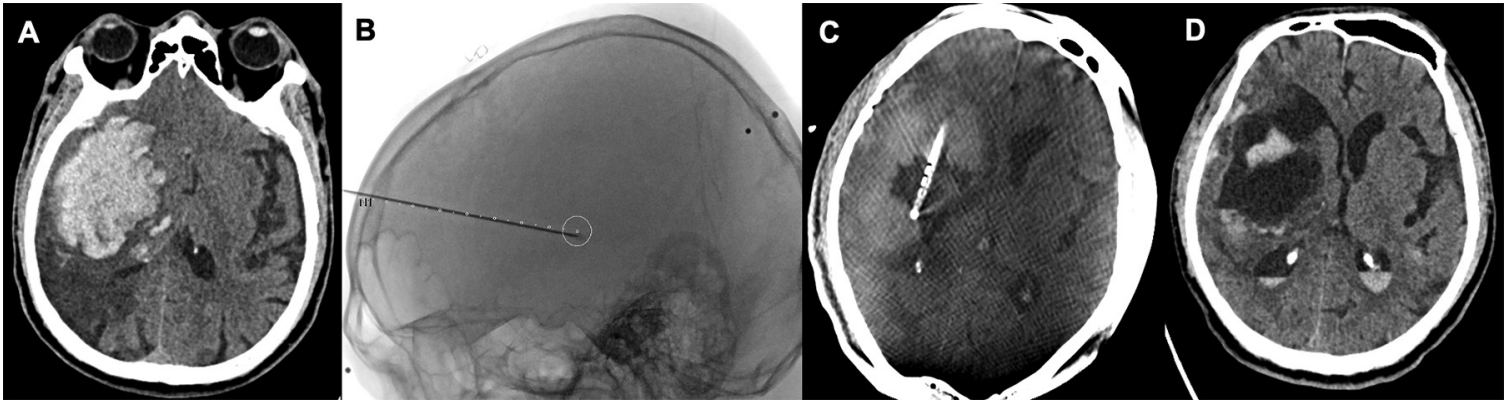

Figure 1 (A) Non-contrast head CT (axial) demonstrating a large right basal ganglia hemorrhage with significant mass effect. (B) Ventricular drain stylet overlaid on trajectory planning using flat panel detector CT-based navigation assistance for minimally invasive surgery intracerebral hemorrhage (ICH) evacuation. (C) Placement of drain following evacuation with interval decrease in the intraparenchymal hemorrhage volume prior to tissue plasminogen activator (tPA) administration. (D) Non-contrast head CT (axial) demonstrating significant improvement in the right basal ganglia hemorrhage following ICH evacuation and Minimally Invasive Surgery and tPA in Intracerebral Hemorrhage Evacuation (MISTIE) protocol.

in the interventional arms of ongoing clinical trials, including MIND, INVEST-FEASIBILITY, and ENRICH. ${ }^{9} 101819$

In addition to further development and evolution of new MIS techniques, the use of real-time advanced neuroimaging and navigation techniques (syngo iGuide needle guidance software, Siemens Healthineers, Forchheim, Germany; XperCT, XperGuide, Philips Healthcare, Best, the Netherlands) provides a synergistic opportunity to minimize tissue manipulation during hematoma evacuation in the interventional suite. In this paper, we describe the use of angiosuite-based MIS hematoma evacuation with the use of flat panel detector computed tomographic imaging.

\section{CASE ILLUSTRATIONS}

\section{Case 1}

A patient in their $70 \mathrm{~s}$ with a history of hypothyroidism, hypertension, and prior right middle cerebral artery stroke due to atrial fibrillation (on warfarin) presented with mild disorientation, somnolence, left hemineglect, and severe left hemiparesis. The patient underwent a non-contrast head CT, which identified a large right basal ganglia ICH exhibiting significant mass effect (figure 1). ICH score was 2 for both volume $(88 \mathrm{cc})$ and intraventricular extension. Volume and intraventricular extension were initially medically managed with reversal of the patient's coagulopathy, hypertonic saline, and close neuromonitoring. Unfortunately, the patient continued to experience worsening somnolence, and the decision was made to proceed with intervention. The patient was subsequently taken to the angiography suite, and an $\mathrm{ICH}$ evacuation with the assistance flat panel detector CT (FDCT)-based navigation was performed using the MISTIE technique. After 72 hours of treatment, the catheter was ultimately removed, recovery continued, and a follow-up non-contrast head CT revealed significant improvement in $\mathrm{ICH}$ volume and mass effect. The patient was ultimately discharged to a skilled nursing facility and has progressed to living at home, although remaining dependent on family for care.

\section{Case 2}

A patient in their 60 s with a history of hypertension, hyperlipidemia, and coronary artery disease on $81 \mathrm{mg}$ aspirin presented after sudden onset of right hemiplegia, right neglect, mild aphasia, and drowsiness. A non-contrast head CT scan revealed a large, left basal ganglia and temporoparietal intraparenchymal hemorrhage with intraventricular extension (figure 2). A CT angiogram revealed no evidence of underlying vascular malformation. On the second hospitalization day, the patient's somnolence progressed and they were subsequently brought to the interventional suite. A FDCT image was obtained in the angiography suite and transferred to a traditional neuronavigation platform. A trajectory was then planned along the long axis of the hematoma. A peel-away sheath was then placed under traditional navigation guidance (figure 3 ). With the sheath in place, MIS evacuation of the hematoma was started using the Stereotactic Intracerebral Hemorrhage Underwater Blood Aspiration (SCUBA) technique, with adequate hematoma evacuation confirmed with an intraprocedural FDCT. Follow-up noncontrast head CT demonstrated good evacuation of the hemorrhage. The patient recovered well and was discharged to a skilled

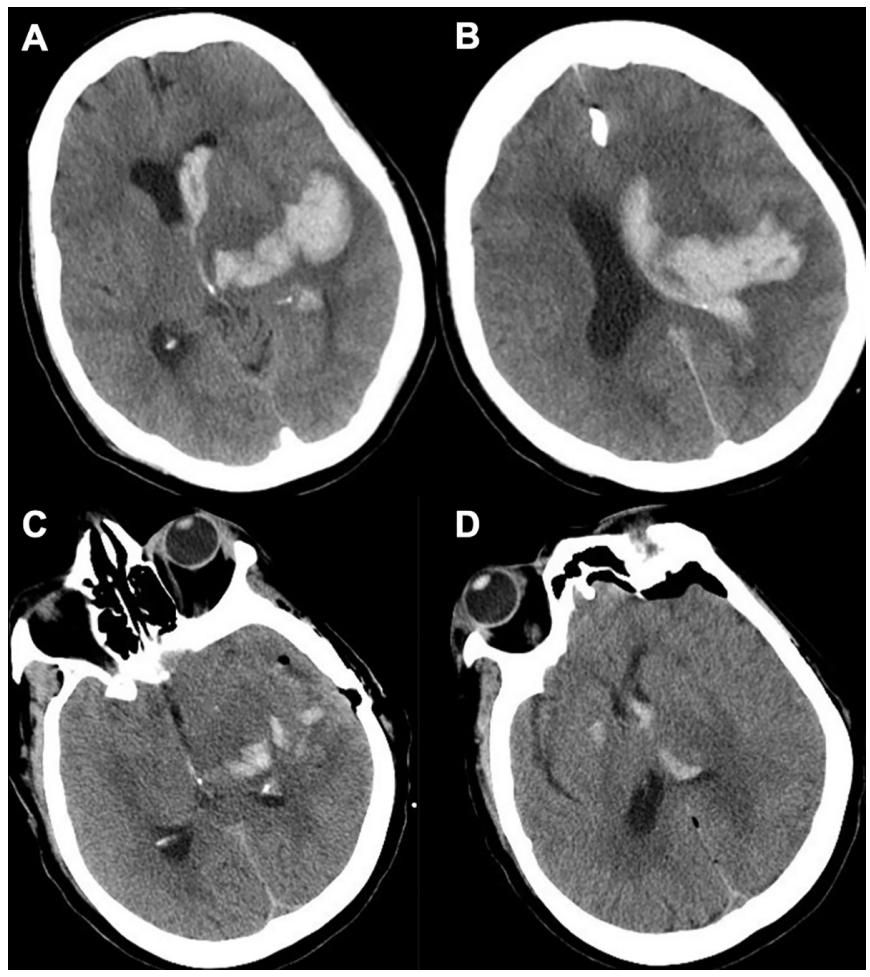

Figure 2 (A, B) Non-contrast head CT (axial) demonstrating a left basal ganglia and temporoparietal intraparenchymal hemorrhage with intraventricular extension with mild mass effect and midline shift. (C, D) Non-contrast head CT (axial) demonstrating significant improvement in the hemorrhage following intracerebral hemorrhage evacuation with the Stereotactic Intracerebral Hemorrhage Underwater Blood Aspiration (SCUBA) protocol. 


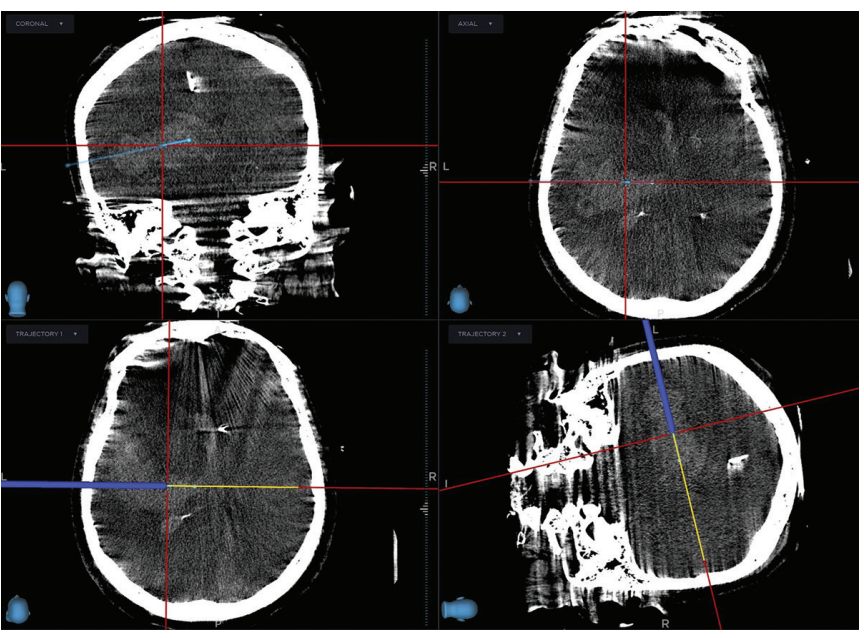

Figure 3 After obtaining flat panel detector CT images with syngo workstation reconstruction (Siemens, Erlangen, Germany), the images are transferred to a stealth navigation system (Medtronic, Minneapolis, Minnesota, USA), and a peel-away sheath is placed under traditional navigation guidance.

nursing facility. At 90 days postprocedure, the patient was living at home with mild deficits (modified Rankin score 2).

\section{CASE SELECTION}

The use of FDCT-based navigation for assistance with MIS hematoma evacuation occurs regularly in the interventional suite and is best used for lobar, basal ganglia, or deep white matter tract hematomas. Patients with hematomas causing significant midline shift, brain herniation, or with significant surrounding cerebral edema requiring full decompressive hemicraniectomy would generally be best suited for surgical evacuation in the operating room.

Conventional CT is used as the primary diagnostic and surveillance tool and aids in determining clot characteristics for MIS evacuation eligibility. Additionally, CT angiography and/or digital subtraction angiography is acquired before proceeding with hematoma evacuation to assess for other hemorrhagic lesions, such as underlying vascular malformations or tumors that might require alternative management strategies in addition to minimally invasive hematoma evacuation. This is of particular importance if MISTIE or SCUBA techniques are limited as options to mitigate active hemorrhage during the procedure. ${ }^{1720}$ The use of a minimally invasive parafascicular surgical (MIPS) approach—as often enabled by MR—can be used in the setting of an active spot sign or underlying lesion since the use of a port allows additional flexibility. ${ }^{11} 1219$ Regardless of the ultimate technical approach, high quality CT and angiographic assessment are needed before proceeding with MIS evacuation.

\section{FLAT PANEL DETECTOR CT (FDCT)-ASSISTED NAVIGATION}

Flat panel detector CT-based navigation is an effective, convenient, and efficient option to conventional CT for procedures based in the angiography suite. Modern angiosuite-based FDCT systems (syngo and XperCT) generate comparable quality images of hemorrhage, soft tissue differentiation, and general anatomic relationships, with a $13-42 \%$ lower total radiation dose than conventional $\mathrm{CT}^{21}$ Fine differences in tissue density may be more difficult to detect, but nevertheless FDCT provides submillimeter resolution, which is typically sufficient for identification of intraparenchymal hematomas, given the stark difference in contrast between parenchyma and hemorrhage. Furthermore, integrated navigation software provides the ability to perform percutaneous procedures safely and efficiently and has been employed in the treatment of numerous cranial pathologies, including external ventricular drain placement, sclerotherapy for venous or lymphatic malformations, intramedullary spinal cord infusions, or embolization of dural arteriovenous fistulae. ${ }^{22-26}$

One of the primary disadvantages of FDCT-assisted navigation is difficulty with maintaining patient positioning. Traditional operative navigation uses a head clamp to maintain rigid fixation - an option not available for many interventional suites. Although this can often be overcome by taping a patient's head to minimize patient motion, FDCT-based navigation systems can mitigate motion to a degree through reorientation of navigation images with live fluoroscopy and osseous landmarks. Additionally, if excessive patient motion occurs or there is concern about navigation inaccuracy, the opportunity to obtain new 3D FDCT imaging is readily available without needing to move the patient. Furthermore, automatic motion correction software will adjust for more subtle patient movement, improving navigation accuracy.

FDCT images can also be used for navigation purposes for lesions that are best visualized on MR imaging. Newer software allows the user to fuse preprocedure MRI with FDCT imaging obtained at the time of the procedure to enable navigation of targets not optimally visualized with $\mathrm{CT}^{27}{ }^{28}$ Previous studies assessing the accuracy of targeting deep lesions only visualized on MR imaging and using FDCT-based navigational software found this method to be highly accurate with a radial error of 2.5-3.0 mm, a similar rate to lesions targeted solely with CT without fusion overlay. ${ }^{24}$

Importantly, FDCT-based navigation has been established to accurately target intracranial lesions within $2-4 \mathrm{~mm},{ }^{2124} 29$ and previous experience with FDCT for ICH evacuation has been observed to be safe, reliable, and convenient. The use of FDCTbased navigation in patients with supratentorial hypertensive $\mathrm{ICH}$ has been shown to reliably reduce a significant degree of ICH burden and also improve clinical neurologic function. ${ }^{29}$ Patients exhibited improved in-hospital neurologic function and short-term clinical recovery following discharge. Although this technology has shown promise to improve the efficiency and efficacy of hematoma evacuation, it remains underused by the neurointerventionalists.

\section{IMAGE PREPARATION FOR FDCT-ASSISTED EVACUATION}

For reference, this manuscript will detail the use of needle guidance for clinical applicability in evacuation of ICH using syngo needle guidance (formally known as syngo iGuide) software (Siemens Healthineers, Forchheim, Germany). Comparable image-guided navigation software is also available on other fixed C-arm systems (XperCT, XperGuide, Philips Inc.). Once in the angiography suite and placed under general anesthesia, the patient is transferred to the angiography table in a supine position. Fiducials may be used (eg, glabella, bilateral tragus, and bilateral lambdoid sutures) to improve navigation accuracy, but these are not essential as one can use bony and external landmarks to assess navigation accuracy. Once positioned, a $20 \mathrm{~s}$ DynaCT head or comparable scan should be performed $(109 \mathrm{kV}$, Hounsfield unit normal kernel, with an isotropic resolution of $0.46 \mathrm{~mm}$ and (if available) motion compensation algorithms and metal artifact reduction features). The scan allows visualization of the hematoma, registration of the navigation system to the patient, and provides a platform for identification of an appropriate entry point, trajectory, and target. 


\section{Procedure}

After obtaining images and trajectory planning, FDCT images can be used in a variety of ways. When using MISTIE or SCUBA techniques, we tend to use syngo needle guidance for assistance with the entry point and trajectory of catheter placement. Given the additional equipment needed for either SCUBA or MIPS techniques, however, it might be advantageous to use traditional neuronavigation software as it affords greater freedom of movement around the patient's head without interference from the $\mathrm{C}$-arm. This does require transferring FDCT images to a separate navigation system, but these can be readily fused to preoperative imaging and is a relatively quick process. Importantly, it allows navigation to be performed on real-time images and allows the opportunity to rescan the patient quickly and easily, if there is concern about navigation accuracy, and to assess the quality of evacuation during the procedure.

\section{Traditional guidance using neuronavigation software}

In order to transfer FDCT imaging to traditional neuronavigation software, the C-arm is backed out after completion of $3 \mathrm{D}$ FDCT acquisition. The table can also be rotated to allow optimal space for operator maneuverability during the procedure. Imaging data can then be transferred to a separate navigation system with either a USB, CD, or through a hospital network node. Once the data file has been transferred, an entry point and trajectory can be set using FDCT imaging with or without fusion to a preoperative MR image. The patient is then registered to navigation using classic external landmarks. As mentioned previously, this is ideal when using a SCUBA or MIPS approach for minimally invasive hematoma evacuation.

Once the patient is registered, the skin can be prepped, lidocaine administered, and a $1-2 \mathrm{~cm}$ incision made over the entry point. A burr hole is made with a high-speed electric drill and perforator drill bit, and the dura is opened sharply. Using the set trajectory, a $19 \mathrm{~F}$ peel-away sheath can be advanced to the depth of the hematoma under navigation guidance. The inner stylet is removed, and a tri-port rigid endoscope is advanced through the sheath into the hematoma. A combination of irrigation, aspiration, and hematoma agitation can then be used to remove the hematoma under direct endoscopic visualization. Similarly, if a MIPS approach is used, a small trephine craniotomy allows for trans-sulcal dissection and placement of a port into the hematoma under FDCT navigation guidance, allowing hematoma evacuation through either exoscope or microscope visualization. It is important to note that using FDCT to augment traditional navigation guidance would typically require a hybrid suite, given the additional equipment and structure of most angiography suites, which substantially limits its applicability. The primary benefit of using FDCT navigation guidance in this manner is to allow guidance based on real-time imaging and to allow for repeat scans to be obtained at the end of the procedure.

\section{Neuronavigation software}

The syngo needle guidance program (also referred to as syngo iGuide) allows for direct neuronavigation without needing to transfer FDCT imaging data to a separate navigation platform. After the spin and 3D head reconstruction is complete, the volume can be windowed to optimize the visualization of the hematoma and surrounding parenchymal tissue. After volume preparation, needle guidance workflow is used to find and select desirable entry and target points. The planned needle trajectory and path can be further analyzed to avoid eloquent tissue, plan the trajectory through the long axis of the hematoma, and ensure
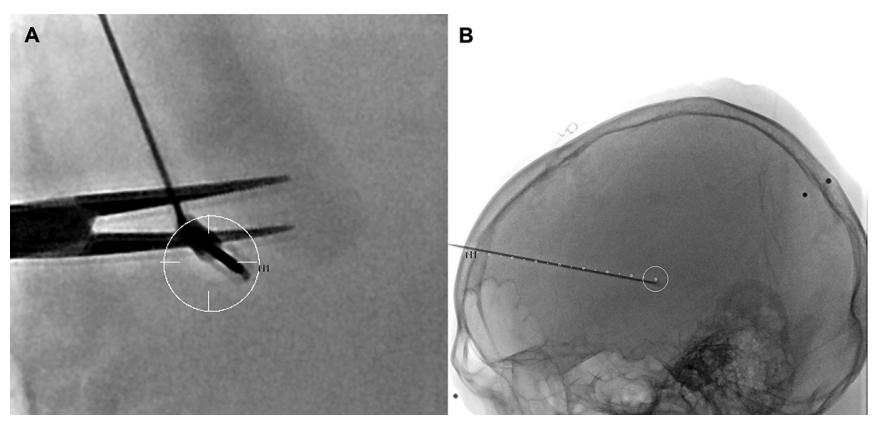

Figure 4 (A) Fluoroscopic image assessing trajectory of catheter in comparison with needle guidance trajectory to target lesion prior to catheter placement. (B) Fluoroscopic image of catheter and stylet following placement in an intraparenchymal hematoma and overlaid needle guidance trajectory and target.

a comfortable working angle for the surgeon. Prior MR images or conventional CT images can also be imported at this stage and fused with the obtained FDCT imaging to facilitate safe trajectory planning.

With the trajectory set, the angiography table is floated for table positioning, and integrated laser crosshairs identify the entry point, which can be confirmed with fluoroscopy (online supplemental appendix). It can be helpful to rotate the table to an oblique position to allow better access. At this point, a $1-2 \mathrm{~cm}$ incision is made, a burr hole fashioned with the high-speed electric drill, and the dura opened sharply. The table should then be repositioned to midline to allow use of the FDCT navigational guidance. New fluoroscopic 2D images can then be overlaid on volume rendering technique images for automatic registration and the table can be manually shimmed to align the volume rendering technique and 2D images. While bony landmarks are sufficient for this step, fiducials can provide additional assistance in aligning the FDCT images with the patient. If automatic registration and alignment is not sufficient, a manual adjustment can be made.

Fluoroscopy can be used to confirm that the burr hole is appropriately located at the planned entry point. If needed, a new trajectory and entry point can be obtained on the workstation based on the burr hole location. Using a long clamp to hold the external ventricular drain (EVD) catheter at the entry site, fluoroscopy is used to match the laser crosshairs with the trajectory of the catheter (figure 4A). With the crosshairs aligned, the clamp is removed and the catheter is delivered in-line with the laser crosshairs to the specified depth (figure 4B). Catheter position can be verified with a shorter $5 \mathrm{~s}$ DR head DynaCT $(70$ $\mathrm{kV}$, Hounsfield unit normal kernel, with an isotropic resolution of $0.46 \mathrm{~mm}$ ) or similar acquisition. Once in position, the stylet is removed and aspiration is performed with a $10 \mathrm{cc}$ syringe, removing hematoma until resistance is encountered. Before concluding the procedure, a $20 \mathrm{~s}$ Dyna CT scan is performed (using the same parameters as the prior FDCT) to quantify the hematoma evacuation. If satisfactory, the catheter is tunneled, connected to an EVD drainage catheter, and the incision is closed.

\section{INTRAOPERATIVE FDCT IMAGING}

The use of real-time FDCT imaging obtained immediately before and after the procedure has significant advantages given potential hematoma expansion. More importantly, the ability to obtain additional 3D imaging quickly and readily during the procedure is perhaps the most important advantage of this 
method. A volumetric FDCT scan can be obtained as needed without moving the patient to assess the degree of ICH evacuation, catheter placement, or navigation accuracy.

Previous studies using intraoperative CT or cone-beam CT imaging immediately following initial hematoma evacuation have led to further hematoma evacuation in a third of these patients, ultimately leading to overall improved hematoma evacuation. ${ }^{30}$ This opportunity to assess surgical performance and the extent of evacuation at the time of surgery allows for a greater percentage of hematoma reduction, which has been linked with improved patient outcomes. ${ }^{10}$ It is our practice to obtain a FDCT scan any time there is significant concern about navigation accuracy, and also at the end of the procedure to assess the overall percentage of evacuation and residual hematoma volume. In instances with substantial residual hematoma, further evacuation can start, or the catheter trajectory can be repositioned, if necessary.

\section{POTENTIAL PITFALLS AND CHALLENGES}

While the use of FDCT imaging can be used in any minimally invasive approach to intraparenchymal hematoma evacuation, syngo needle guidance software is ideally used for placement of either an EVD catheter (MISTIE approach) or a 19F peel-away sheath (SCUBA technique). The positioning and movement of the $\mathrm{C}$-arm during navigational guidance can be prohibitive by limiting surgeon movement and make positioning for catheter placement difficult. It is also important to note that given the lack of rigid head fixation and limitations with patient positioning, using this approach for a posterior approach is difficult and probably best avoided. Furthermore, the surgeon will invariably get a degree of radiation exposure to their hands while maintaining an appropriate trajectory when passing the catheter into the hematoma, although the biological effects of this limited radiation exposure are minimal. ${ }^{31}$

The primary pitfall associated with the use of FDCT-based navigation is the loss of accuracy and potential for catheter misplacement and poor hematoma evacuation. In the absence of a rigid radiolucent head clamp, the head should be firmly secured with tape before obtaining the initial FDCT images. With adequate tape securing the head, particularly for neutral head positioning, navigation accuracy tends to remain reliable throughout the procedure. Additional head rotation and patient manipulation, however, increases the likelihood of navigation accuracy loss and may prompt several additional repeat images to be obtained. Navigation accuracy should certainly be checked as suitable before draping and before catheter placement, with external landmarks as points of reference. If any concerns arise pertaining to navigation accuracy, a new FDCT image should be obtained prior to further catheter placement or manipulation.

\section{CONCLUSION}

Acute interventions for intracerebral hemorrhage are critical to minimize primary and secondary brain injury from hematoma and related sequelae. With ongoing advances in minimally invasive procedures for intraparenchymal hematoma evacuation, needle guidance software provides a unique opportunity for the interventional surgeon. The ability to use real-time imaging for navigation guidance and to assess the quality of evacuation intraprocedurally are particularly advantageous, especially in light of growing evidence supporting the importance of hematoma evacuation completeness. Minimally invasive procedures for hematoma evacuation have the potential to augment acute management strategies and improve patient outcomes.

\section{Author affiliations}

${ }^{1}$ Department of Neurosurgery, University of Kentucky, Lexington, Kentucky, USA ${ }^{2}$ Department of Neurosurgery, University of Tennessee Health Science Center, Memphis, Tennessee, USA

${ }^{3}$ Department of Neurosurgery, Semmes-Murphey Neurologic and Spine Institute, Memphis, Tennessee, USA

${ }^{4}$ Department of Neurology, University of California San Francisco, San Francisco, California, USA

${ }^{5}$ Department of Neurology, Weill Institute for Neurosciences, University of California San Francisco, San Francisco, California, USA

${ }^{6}$ Department of Research and Development, Siemens Medical Solutions USA Inc, Malvern, Pennsylvania, USA

${ }^{7}$ Department of Neurosurgery, Stony Brook University, Stony Brook, New York, USA ${ }^{8}$ Department of Neurosurgery, SUNY SB, New York, New York, USA

${ }^{9}$ Department of Radiology and Biomedical Imaging, University of California San Francisco, San Francisco, California, USA

Correction notice Since this article was first published online, Dr Cathra Halabi's conflicts of interest have been updated to none to declare.

Twitter David Dornbos III @DornbosIII_MD and Adam S Arthur @AdamArthurMD

Acknowledgements The authors would like to thank Andrew J Gienapp (Neuroscience Institute, Le Bonheur Children's Hospital, Memphis, Tennessee and Department of Neurosurgery, University of Tennessee Health Science Center, Memphis, Tennessee) for copy editing, preparation of the manuscript and figures for publishing, and publication assistance.

Contributors All authors contributed to the conception and design; acquisition, analysis, or interpretation of data; and drafting and critically revising the manuscript.

Funding The authors have not declared a specific grant for this research from any funding agency in the public, commercial or not-for-profit sectors.

Competing interests $\mathrm{CH}$ has no competing interests to report. JD, KM are employees of Siemens Medical Solutions. DF is a consultant for Arsenal Medical, Balt USA, Cerenovous, Marblehead, Medtronic, MENTICE-Vascular Simulations, Microvention, Neurogami, Qapel Medical, RAPID Medical, RAPID.Al, Stryker; received research support from Balt USA, Microvention, Penumbra, Siemens, and Stryker; has received honorarium from Qapel Medicine; is a stockholder in Marblehead, MENTICE-Vascular Simulations, and Neurogami; and a member of the Journal of Neurolnterventional Surgery editorial board. DLC has no competing interests to report. ASA is a consultant for Balt, Johnson and Johnson, Medtronic, Microvention, Penumbra, Scientia, Serenity, and Stryker; and received research support from Balt USA, Medtronic, Microvention, Penumbra, and Siemens. DD is a consultant for Imperative Care and has received research suport from Siemens.

Patient consent for publication Not applicable.

Ethics approval Institutional review noard approval was not obtained as this study is a review of an existing imaging technology. Patient images and descriptions featured in the manuscript have been completely anonymized to ensure patient privacy.

Provenance and peer review Not commissioned; externally peer reviewed Supplemental material This content has been supplied by the author(s). It has not been vetted by BMJ Publishing Group Limited (BMJ) and may not have been peer-reviewed. Any opinions or recommendations discussed are solely those of the author(s) and are not endorsed by BMJ. BMJ disclaims all liability and responsibility arising from any reliance placed on the content. Where the content includes any translated material, BMJ does not warrant the accuracy and reliability of the translations (including but not limited to local regulations, clinical guidelines, terminology, drug names and drug dosages), and is not responsible for any error and/or omissions arising from translation and adaptation or otherwise.

Open access This is an open access article distributed in accordance with the Creative Commons Attribution Non Commercial (CC BY-NC 4.0) license, which permits others to distribute, remix, adapt, build upon this work non-commercially, and license their derivative works on different terms, provided the original work is properly cited, appropriate credit is given, any changes made indicated, and the use is non-commercial. See: http://creativecommons.org/licenses/by-nc/4.0/.

\section{ORCID iDs}

David Dornbos III http://orcid.org/0000-0002-0039-0016

David Fiorella http://orcid.org/0000-0002-2677-8780

Adam S Arthur http://orcid.org/0000-0002-1536-1613

\section{REFERENCES}

1 Qureshi Al, Mendelow AD, Hanley DF. Intracerebral haemorrhage. Lancet 2009;373:1632-44. 
2 Fiorella D, Arthur A, Bain M, et al. Minimally invasive surgery for intracerebral and intraventricular hemorrhage: rationale, review of existing data and emerging technologies. Stroke 2016;47:1399-406.

3 van Asch CJ, Luitse MJ, Rinkel GJ, et al. Incidence, case fatality, and functional outcome of intracerebral haemorrhage over time, according to age, sex, and ethnic origin: a systematic review and meta-analysis. Lancet Neurol 2010;9:167-76.

4 Hemphill JC, Greenberg SM, Anderson CS, et al. Guidelines for the management of spontaneous intracerebral hemorrhage: a guideline for healthcare professionals from the American Heart Association/American Stroke Association. Stroke 2015;46:2032-60.

5 Poon MTC, Fonville AF, Al-Shahi Salman R. Long-term prognosis after intracerebral haemorrhage: systematic review and meta-analysis. J Neurol Neurosurg Psychiatry 2014;85:660-7.

6 Powers WJ, Rabinstein AA, Ackerson T, et al. Guidelines for the early management of patients with acute ischemic stroke: 2019 update to the 2018 guidelines for the early management of acute ischemic stroke: a guideline for healthcare professionals from the American Heart Association/American Stroke Association. Stroke 2019;50:e344-418.

7 Mendelow AD, Gregson BA, Fernandes HM, et al. Early surgery versus initial conservative treatment in patients with spontaneous supratentorial intracerebral haematomas in the International surgical trial in intracerebral haemorrhage (STICH): a randomised trial. Lancet 2005;365:387-97.

8 Mendelow AD, Gregson BA, Rowan EN, et al. Early surgery versus initial conservative treatment in patients with spontaneous supratentorial lobar intracerebral haematomas (STICH II): a randomised trial. Lancet 2013;382:397-408.

9 Scaggiante J, Zhang $X$, Mocco J, et al. Minimally invasive surgery for intracerebral hemorrhage. Stroke 2018;49:2612-20.

10 Kellner CP, Song R, Pan J, et al. Long-term functional outcome following minimally invasive endoscopic intracerebral hemorrhage evacuation. J Neurointerv Surg 2020;12:489-94.

11 Bauer AM, Rasmussen PA, Bain MD. Initial single-center technical experience with the BrainPath system for acute intracerebral hemorrhage evacuation. Oper Neurosurg 2017:13:69-76.

12 Suijiantarat $\mathrm{N}$, Tecle NE, Pierson $\mathrm{M}$, et al. Trans-sulcal endoport-assisted evacuation of supratentorial intracerebral hemorrhage: initial single-institution experience compared to matched medically managed patients and effect on 30-day mortality. Oper Neurosurg 2018;14:524-31.

13 Vespa P, Hanley D, Betz J, et al. ICES (intraoperative stereotactic computed tomography-guided endoscopic surgery) for brain hemorrhage: a multicenter randomized controlled trial. Stroke 2016;47:2749-55.

14 Troiani Z, Ascanio LC, Yaeger KA, et al. Minimally invasive surgiscopic evacuation of intracerebral hemorrhage. J Neurointerv Surg 2021;13:400.

15 Zhou X, Chen J, Li Q, et al. Minimally invasive surgery for spontaneous supratentorial intracerebral hemorrhage: a meta-analysis of randomized controlled trials. Stroke 2012:43:2923-30.
16 Xia Z, Wu X, Li J, et al. Minimally invasive surgery is superior to conventional craniotomy in patients with spontaneous supratentorial intracerebral hemorrhage: a systematic review and meta-analysis. World Neurosurg 2018;115:266-73.

17 Hanley DF, Thompson RE, Rosenblum M, et al. Efficacy and safety of minimally invasive surgery with thrombolysis in intracerebral haemorrhage evacuation (MISTIE III): a randomised, controlled, open-label, blinded endpoint phase 3 trial. Lancet 2019;393:1021-32

18 Vitt JR, Sun C-H, Le Roux PD, et al. Minimally invasive surgery for intracerebral hemorrhage. Curr Opin Crit Care 2020;26:1-36.

19 Labib MA, Shah M, Kassam AB, et al. The safety and feasibility of image-guided BrainPath-mediated transsulcul hematoma evacuation: a multicenter study. Neurosurgery 2017;80:515-24.

20 Kellner CP, Chartrain AG, Nistal DA, et al. The Stereotactic Intracerebral Hemorrhage Underwater Blood Aspiration (SCUBA) technique for minimally invasive endoscopic intracerebral hemorrhage evacuation. J Neurointerv Surg 2018;10:771-6.

21 Cooke DL, Levitt MR, Kim LJ, et al. Laser-assisted flat-detector CT-guided intracranial access. Int J Comput Assist Radio/ Surg 2016;11:467-72.

22 Cooke DL, Levitt M, Kim LJ, et al. Transcranial access using fluoroscopic flat panel detector CT navigation. AJNR Am J Neuroradiol 2011;32:E69-70.

23 Diaz OM, Toledo MM, Roehm JOF, et al. Unique percutaneous direct puncture technique for occlusion of a hypoglossal canal dural arteriovenous fistula. $J$ Neurointerv Surg 2018;10:1179-82.

24 Mabray MC, Datta S, Lillaney PV, et al. Accuracy of flat panel detector CT with integrated navigational software with and without MR fusion for single-pass needle placement. J Neurointerv Surg 2016;8:731-5.

25 Maroda AJ, Beckmann NA, Sheyn AM, et al. Trimodal embolization of juvenile nasopharyngeal angiofibroma with intracranial extension. Int J Pediatr Otorhinolaryngol 2020;130:109805.

26 Fiorella D, Peeling L, Denice CM, et al. Integrated flat detector CT and live fluoroscopic-guided external ventricular drain placement within the neuroangiography suite. J Neurointerv Surg 2014;6:457-60.

27 Cooke DL, Levitt M, Kim LJ, et al. Intraorbital access using fluoroscopic flat panel detector CT navigation and three-dimensional MRI overlay. J Neurointerv Surg 2010;2:249-51.

28 Levitt MR, Vaidya SS, Su DK, et al. The "triple-overlay" technique for percutaneous diagnosis and treatment of lesions of the head and neck: combined three-dimensional guidance with magnetic resonance imaging, cone-beam computed tomography, and fluoroscopy. World Neurosurg 2013;79:509-14.

29 Yang Z, Hong B, Jia Z, et al. Treatment of supratentorial spontaneous intracerebral hemorrhage using image-guided minimally invasive surgery: initial experiences of a flat detector CT-based puncture planning and navigation system in the angiographic suite. AJNR Am J Neuroradiol 2014;35:2170-5.

30 Hecht N, Czabanka M, Kendlbacher P. Intraoperative CT and cone-beam CT imaging for minimally invasive evacuation of spontaneous intracerebral hemorrhag. Acta Neurochir 2020;162:3167-77.

31 Wagner LK, Eifel PJ, Geise RA. Potential biological effects following high X-ray dose interventional procedures. J Vasc Interv Radiol 1994;5:71-84. 